\title{
Assessment of the ITER electron cyclotron upper launcher capabilities in view of an optimized design
}

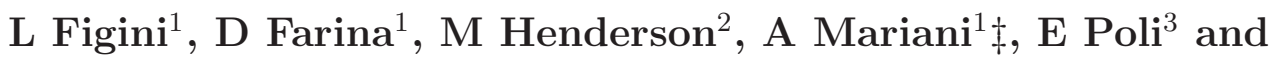 \\ G Saibene ${ }^{4}$ \\ ${ }^{1}$ Istituto di Fisica del Plasma, CNR, 20125 Milano, Italy \\ 2 ITER Organization, 13108 Saint Paul lez Durance, France \\ ${ }^{3}$ Max-Planck-Institut für Plasmaphysik, D-85748 Garching, Germany \\ ${ }^{4}$ Fusion for Energy, E-08019 Barcelona, Spain \\ E-mail: figini@ifp.cnr.it
}

\begin{abstract}
The 24 MW ITER Electron Cyclotron (EC) Heating and Current Drive (H\&CD) system, operating at $170 \mathrm{GHz}$, consists of one Equatorial and four Upper Launchers. The main task of the UL will be the control of Magneto-Hydrodynamic activity such as Neoclassical Tearing Modes (NTMs) at the $q=3 / 2$ and $q=2$ surfaces and sawteeth at $q=1$, but it will also be needed for current profile tailoring in advanced scenarios and to assist plasma break-down and L- to H-mode transition. Moreover, it is required to be effective both when ITER will operate at nominal and reduced magnetic field magnitude.

Here the performance of the Upper Launcher has been assessed through the study of the full temporal evolution of different scenarios, including the reference ITER 15 MA H-mode plasma, a half-field case at $2.65 \mathrm{~T}$, and a steady state scenario. The ECCD efficiency has been evaluated for a wide range of injection angles, deriving the optimal angles and the power required for NTMs stabilization with simplified criteria. An injected power ranging from $3 \mathrm{MW}$ to $9 \mathrm{MW}$ should be sufficient to control NTMs in the flat-top phase of the scenarios considered here. The result of the analysis shows that the EC system maintains a good performance level even at intermediate values of the magnetic field, between the nominal and the half-field value. The analysis has also allowed to evaluate the adequateness of the available steering range for reaching the rational surfaces during all the phases of the discharge, and to quantify the steering sensitivity to shifts of the target or aiming errors. The result is an assessment of the UL design requirements to achieve the desired functionalities, which will be used to drive the optimization and finalization of the UL design.
\end{abstract}

PACS numbers: $52.35 . \mathrm{Hr}, 52.50 . \mathrm{Sw}, 52.55 . \mathrm{Wq}$

Keywords: Heating and current drive, electron cyclotron waves, ITER

Submitted to: Plasma Phys. Control. Fusion

$\ddagger$ Present address: Ecole Polytechnique Fédérale de Lausanne, Centre de Recherches en Physique des Plasmas, CH-1015 Lausanne, Switzerland 


\section{Introduction}

The Electron Cyclotron (EC) Heating and Current Drive (H \& CD) system in ITER [1], operating at $170 \mathrm{GHz}$, will deliver up to $20 \mathrm{MW}$ of heating power to the plasma. Among the planned additional heating systems, it will be the first to operate and the only one foreseen for the first ITER plasma [2]. The system has been assigned a list of functions, which include central heating, bulk current drive, the control of MagnetoHydrodynamic (MHD) activity such as Neoclassical Tearing Modes (NTMs) at the $q=3 / 2$ and $q=2$ surfaces and sawtooth (ST) instabilities at the $q=1$ surface, the assist to break-down and L-mode to H-mode transition phases of the plasma discharge. These functions have been partitioned between one Equatorial Launcher (EL) located close to the equatorial plane of the plasma and four Upper Launchers (UL) injecting EC waves from the upper ports [2]. The two types of launcher have been designed with different tasks in mind, assigning to the EL the function of supporting central heating and bulk current drive, and to the UL the control of NTMs. In order to fulfil these main functions, the characteristics of the launchers have been selected on the basis of different plasma scenarios, maximizing the current driven from the EL, with broad absorption profiles in the inner plasma region, and aiming for large peak current density and localized absorption in the outer radial range for the UL, using beams focussed close to the resonant layer in order to minimize the absorption volume [3, 4].

According to the present design, four identical UL will house eight beams each, split in two groups of four. The grouped beams follow a similar optical path and share the final steering mirror. The two steering mirrors in the launcher, independently movable, are positioned at different heights in the upper port plug, and they are identified as Upper Steering Mirror (USM) and Lower Steering Mirror (LSM). The steering mechanism rotates the mirror around an axis, which mainly varies the poloidal injection angle, keeping the toroidal injection angle almost constant. Finally, a switching system can deviate the 24 beams coming from the gyrotrons to a number of combinations of the 16 entrances associated with the USM and the 16 associated with the LSM. Therefore, accounting for the transmission losses, the maximum power that can be injected from each set of steering mirrors is $P_{\mathrm{EC}} \simeq 13.3 \mathrm{MW}$, out of a total of $20 \mathrm{MW}$.

This paper presents the recent analysis done to assess the potential of EC wave injection from the UL through the evaluation of the heating and current drive performance in a set of plasma scenarios, at nominal and reduced magnetic field strength, and considering the full temporal evolution of the discharge. The purpose here is to gain a clearer overview of the behaviour of the EC system over a range of conditions as wide as possible, in order to drive the design of the launcher and to help in its refinement to achieve all the foreseen functionalities, similarly to what has already been done for the Equatorial Launcher $[5,6]$.

The modelling framework adopted throughout the following analysis is described in section 2, introducing the simulation tools, the definitions and the criteria used. The main features of the plasma scenarios on which the following analysis is based 
are presented in section 3, and the results obtained are discussed in section 4 with an assessment of the ECCD efficiency, an evaluation of the power required to stabilize NTMs at the $q=3 / 2$ and $q=2$ surfaces, and an estimate of the performance achievable at intermediate values of the magnetic field. The steering range necessary to reach the rational surfaces at different times in the plasma discharge is also computed, as well as the accuracy required to avoid misalignment between the beam and the target or among the beams. Conclusions are drawn in section 5 .

\section{Modelling framework}

The propagation of EC waves in ITER in the form of Gaussian beams, like those foreseen to be injected from the UL, involves quite different scale lengths: the plasma minor radius $a=2 \mathrm{~m}$ which is the typical length over which the plasma properties change, the beam size $w$ designed to be of the order of a few centimeters, and the wavelength $\lambda \simeq 1.8 \mathrm{~mm}$ for a frequency $f=170 \mathrm{GHz}$. A clear ordering $\lambda \ll w \ll a$ is thus always maintained, also in the plasma where the typical density $n_{\mathrm{e}} \sim 10^{20} \mathrm{~m}^{-3}$ is much lower than the cut-off density $\left(n_{\mathrm{e}, \mathrm{co}}=3.5810^{20} \mathrm{~m}^{-3}\right.$ for O-mode), and asymptotic techniques like beam tracing or complex geometrical optics can be used to model the wave propagation $[7,8]$.

Given the large plasma volume, the EC absorbed power density remains moderate even for narrow deposition profiles, peaking to values of the order of $|\mathrm{d} P / \mathrm{d} V|<$ $0.1 \mathrm{MW} / \mathrm{m}^{3}$ per injected MW. Quasi-linear effects are negligible [9], except possibly during the very initial and final stages of the discharge which are not addressed here. A further consequence of the large size of the device is that good numerical accuracy is required in the modelling of EC wave propagation and absorption to avoid error accumulation along the beam propagation path.

All the calculations of ECH\&CD made for the analysis presented here have been performed with the EC beam-tracing code GRAY [10], which aims at computing the propagation, absorption and current drive of EC Gaussian beams with general astigmatism in a generic 2D axisymmetric tokamak plasma. The code assumes a linear plasma response, wave propagation and beam shape evolution are computed for a cold plasma using the complex eikonal approach to account for diffraction effects, absorption is described with a fully relativistic formulation, and the driven current is evaluated with a model which includes momentum conservation [11] within the linear adjoint approach. The proper implementation of the physics models in the GRAY code and their applicability to ITER plasma scenarios have been verified with several benchmarks against other ray- and beam-tracing codes, both in the framework of an ITPA activity [12], and more recently within the European Integrated Tokamak Modelling Task Force after the addition of momentum conservation in the computation of current drive efficiency [13].

The possibility to handle astigmatic beams implemented in GRAY is an important feature for present and future finer analysis, since general astigmatism is expected for the 
Table 1. Launching coordinates and beam parameters.

\begin{tabular}{lcccc}
\hline Mirror & $R(\mathrm{~m})$ & $z(\mathrm{~m})$ & $w_{0}(\mathrm{~cm})$ & $d_{0}(\mathrm{~m})$ \\
\hline USM & 7.00 & 4.41 & 2.9 & 2.13 \\
LSM & 7.05 & 4.18 & 2.1 & 1.62 \\
\hline
\end{tabular}

beams after their last reflection on the steering mirrors. At present, however, the details of the launcher's optical system have not been fixed yet and the beam shaping is still subject to refinements. Here, for the sake of simplicity, a single stigmatic representative beam has been considered for each of the USM and of the LSM, using the same beam parameters as in [4]. The launching coordinates $R$ and $z$ for each steering mirror correspond to the incidence point on the steering mirror of such a representative virtual beam. The launching coordinates, the beam waist $w_{0}$ and the focal distance $d_{0}$, i.e. the distance between the injection point and the beam waist for propagation in vacuum, are shown in table 1 for the two virtual beams. The actual incidence points of the four beams sharing the same steering mirror lie along the steering axis, evenly distributed on the two sides of the virtual launching point, over a length of about $0.15 \mathrm{~m}$ mainly in toroidal direction. Such a small spatial shift $(\delta R<0.02 \mathrm{~m}, \delta z<0.03 \mathrm{~m})$ does not alter ECCD performance significantly, the main effect being the need to adjust the injection angles by fractions of degree to recover the same results [14]. For the same reason the launching coordinates have been considered constant for varying poloidal and toroidal injection angles.

For both the USM and the LSM, a wide scan around the nominal injection angles have been performed, to gain an overview of the current drive performance and of its variations for deviations from the present optical design. The current design foresees steering the beams in the poloidal direction, with a toroidal injection angle $\beta=\arcsin \left(k_{0, \phi} / k_{0}\right)$ almost constant at $20^{\circ}$ and a variable poloidal injection angle $\alpha=\arctan \left(k_{0, z} / k_{0, R}\right)$, over a range $\Delta \alpha_{\text {steer }} \simeq 24^{\circ}$, being $k_{0, R}, k_{0, \phi}, k_{0, z}$ the components of the wave vector at launch in a cylindrical right-handed $(R, \phi, z)$ reference frame, having the $z$-axis aligned with the tokamak symmetry axis. For the following analysis, the two angles have been varied over the ranges $25^{\circ} \leq \alpha \leq 70^{\circ}$ and $15^{\circ} \leq \beta \leq 25^{\circ}$ respectively, with even steps $\delta \alpha=\delta \beta=1^{\circ}$.

Injection of ordinary mode $(\mathrm{OM})$ polarized waves has been considered for all the scenarios except for the half-field case, for which extraordinary mode (XM) injection has been assumed since XM interaction at the second harmonic is more efficient than OM (see figure 1).

NTM control is one of the main tasks assigned to the UL. It is thus desirable to have a criterium to evaluate the power required for the stabilization of NTMs being simple enough to be used in extensive analysis, and at the same time accurate enough to allow a derivation of quantitative results to drive the launcher's design towards optimum efficiency. The temporal evolution for the size $w$ of an island can be modelled through 


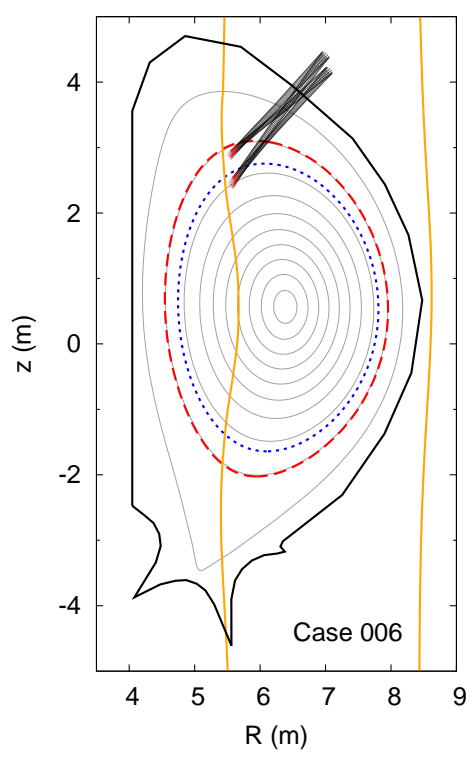

Figure 1. Beam tracing from USM and LSM aiming at the $q=2\left(--_{-}\right)$and $q=3 / 2$ $(\ldots \ldots)$ surfaces for the half-field scenario at $t=820 \mathrm{~s}$. The third and second harmonic cold resonances (yellow vertical lines) are outside the plasma and on the high field side of the magnetic axis respectively.

the generalized Rutherford equation [15], given here in a simplified form [16, 17]:

$$
\frac{\mathrm{d} w}{\mathrm{~d} t} \propto-1+\frac{w_{\mathrm{sat}} w}{w^{2}+w_{\mathrm{marg}}^{2}}-5.05 \frac{w_{\mathrm{cd}} w_{\mathrm{sat}}}{w^{2}} \frac{J_{\mathrm{cd}}}{J_{\mathrm{bs}}} \eta_{\mathrm{cd}},
$$

where $w_{\text {sat }}$ and $w_{\text {marg }}$ are respectively the saturated and the marginal island width, $J_{\mathrm{bs}}$ is the local bootstrap current density, $J_{\text {cd }}$ and $w_{\text {cd }}$ are respectively the peak value and the full width at $1 / e$ of the Gaussian-shaped driven current density profile, and $\eta_{\mathrm{cd}}$ is a stabilization efficiency term depending on the ratio between $w_{\mathrm{cd}}$ and $w$.

Asymptotic expressions of this equation are available in literature [18, 19, 20], derived for driven current profile width much larger or much smaller than the island size, which allow to obtain simplified criteria for the stabilization of a Neoclassical Tearing Mode in such limits. When $w_{\mathrm{cd}} \gg w$, the criterion is expressed in terms of the ratio between the peak value of the current density $J_{\text {cd }}$ driven by the injected EC wave and the bootstrap current density $J_{\mathrm{bs}}$, and for ITER-like parameters reads [18]

$$
\eta_{\mathrm{NTM}}=\frac{J_{\mathrm{cd}}}{J_{\mathrm{bs}}}>1.2,
$$

while for $w_{\text {cd }} \ll w$ most of the current can be driven inside the island, and the criterion relates to the total driven current $\left(\sim J_{\mathrm{cd}} w_{\mathrm{cd}}\right)$, reading

$$
\eta_{\mathrm{NTM}} w_{\mathrm{cd}}>0.05 \mathrm{~m}, \quad \text { for } w_{\mathrm{cd}}<0.05 \mathrm{~m}
$$

for ITER-like parameters. One can note that the two criteria match when $w_{\mathrm{cd}}=$ $0.05 / 1.2 \mathrm{~m} \simeq 0.042 \mathrm{~m}$. Defining $\eta_{\mathrm{NTM}, 1}$ the driven current density ratio of (2) obtained 
Table 2. Timing of the plasma discharge for the five scenarios considered in the analysis.

\begin{tabular}{lllllll}
\hline & Case 1 $(2,3)$ & & Case 6 & Case 8 \\
& $t(\mathrm{~s})$ & $I_{\mathrm{p}}(\mathrm{MA})$ & $t(\mathrm{~s})$ & $I_{\mathrm{p}}(\mathrm{MA})$ & $t(\mathrm{~s})$ & $I_{\mathrm{p}}(\mathrm{MA})$ \\
\hline Start & $1.3(1.3,31.3)$ & 0.5 & 1.5 & 0.5 & 9.8 & 2.2 \\
L-H transition & 80 & 15 & 32.2 & 7.5 & - & - \\
$I_{P}$ flat-top & $80-530$ & 15 & $32.2-1000$ & 7.5 & $70-2750$ & 10 \\
H-L transition & $530(743,530)$ & $15(7,15)$ & 1036 & 3.0 & - & - \\
End & $623(770,582)$ & 0.5 & 1058 & 0.25 & 2950 & 1.4 \\
\hline
\end{tabular}

with an injected power $P=1 \mathrm{MW}$, two values for the power required for NTM stabilization can be obtained from the above expressions:

$$
P \geq P_{\eta}=1.2 \frac{1 \mathrm{MW}}{\eta_{\mathrm{NTM}, 1}}
$$

and

$$
P \geq P_{\eta w}=\frac{0.05 \mathrm{~m}}{w_{\mathrm{cd}}} \frac{1 \mathrm{MW}}{\eta_{\mathrm{NTM}, 1}}
$$

respectively.

The reconstruction of the EC current and power density profiles as computed in ray/beam tracing codes is somewhat arbitrary in specific conditions, e.g. when the beam trajectory is almost tangent to a magnetic surface. In the GRAY code a suitable area/volume preserving procedure is applied to smooth the profiles, providing a proper profile characterization on a chosen radial grid both in the case of Gaussian-like and irregular profiles. In addition, for a more robust estimate, the definition

$$
J_{\mathrm{p}}=\frac{2}{\sqrt{\pi} \Delta \rho} \frac{I_{\mathrm{cd}}}{(d A / d \rho)_{\langle\rho\rangle_{J}}}
$$

for the peak current density has been introduced in this work, that corresponds to the peak value of a Gaussian profile centered at

$$
\langle\rho\rangle_{J}=\frac{\int d A \rho\left|J_{\mathrm{cd}}(\rho)\right|}{\int d A J_{\mathrm{cd}}(\rho)},
$$

with full width $\Delta \rho$ at $1 / e$, total driven current $I_{\mathrm{cd}}$, and a poloidal cross-section of the plasma $A(\rho)$ enclosed by the flux surface at $\rho$. The definitions in (6) and (7) are introduced for consistency with (1) where the current density profile is assumed to have a Gaussian shape. Note that $J_{\mathrm{p}}$ provides the same peak value as the standard procedure whenever the profiles are "regular", while it provides an appropriate Gaussian average value in case of "spiky" profiles. Here and in the rest of the paper we use as flux label the radius $\rho$ being the squared root of the toroidal flux normalized at the plasma edge. 


\section{Plasma scenarios}

In order to have an overview of the expected performance of the EC system in a wide range of conditions, a total of five scenarios [21] have been considered, along their whole time history: (i) the ITER baseline scenario for inductive H-mode DT plasma, 15 MA, $5.3 \mathrm{~T}, \mathrm{Q}=10$ (Case 1); (ii) a variant with the longest possible current ramp-down (Case 2); (iii) a variant with the shortest possible current ramp-up and ramp-down (Case 3); (iv) a half-field He plasma, 7.5 MA, 2.65 T (Case 6); and (v) a steady-state scenario, 10 MA, 5.3 T (Case 8). The time evolution of the plasma parameters is the result of simulations obtained with 1.5D transport codes coupled with free-boundary equilibrium codes [21]. In these simulations the plasma evolves in a semi-predictive way, with only a few prescribed features like plasma shape and volume-averaged density. The purpose of studying Cases 2 and 3 is to assess the impact of different strategies for starting and ending the plasma discharge on the UL capabilities. The analysis of Case 6 is fundamental to have a good knowledge of the EC system behaviour in the first phases of the ITER operations, when it will run with reduced magnetic field strength. Finally, the main tasks of the EC system in advanced scenarios will be bulk CD and current profile tailoring, so that for Case 8 the focus will be more on pure ECCD performance than on MHD control. In fact in this case $q>1$ over the whole radial extent and the low order $q=2$ and $q=3 / 2$ rational surfaces are less harmful than in the standard scenarios, being closer to the plasma center. The different phases of these scenarios are indicated in table 2, including the duration of the current ramps and flat-top, and the time at which the transitions from L-mode to $\mathrm{H}$-mode confinement and viceversa occurs.

The main parameters required for the evaluation of ECCD and NTM stabilization efficiency on the $q=2$ and $q=3 / 2$ surfaces are reported in table 3 and table 4 respectively, for the Cases 1, 6 and 8 at the end of the current flat-top (Cases 2, 3 are identical to Case 1 in that phase). For the same time values figure 2 shows the profiles of electron temperature $T_{\mathrm{e}}$, density $n_{\mathrm{e}}$ and safety factor $q$ in the three scenarios. Looking at tables 3 and 4 and at figure 2, we can already anticipate here that NTM stabilization efficiency will be higher in the half-field scenario with respect to the baseline case, mainly due to the lower value of the bootstrap current $J_{\mathrm{bs}}$, and also to the slightly more favourable $T_{\mathrm{e}} / n_{\mathrm{e}}$ ratio (i.e. to the larger ECCD efficiency). The highest ECCD efficiency can be expected in the steady-state scenario, which shows the largest $T_{\mathrm{e}} / n_{\mathrm{e}}$ ratio, but at the same time more current needs to be driven for NTM stabilization given the large $J_{\mathrm{bs}}$ in this case.

\section{4. $\mathrm{H} \& \mathrm{CD}$ results}

The ECCD performance has been characterized in detail along the full time history of the five scenarios. A wide range of poloidal and toroidal launching angles has been considered to analyze the performance at the end of the current flat-top phase, namely at $t=520 \mathrm{~s}, t=820 \mathrm{~s}$, and $t=2450 \mathrm{~s}$ for the baseline, half-field, and steady-state scenario 

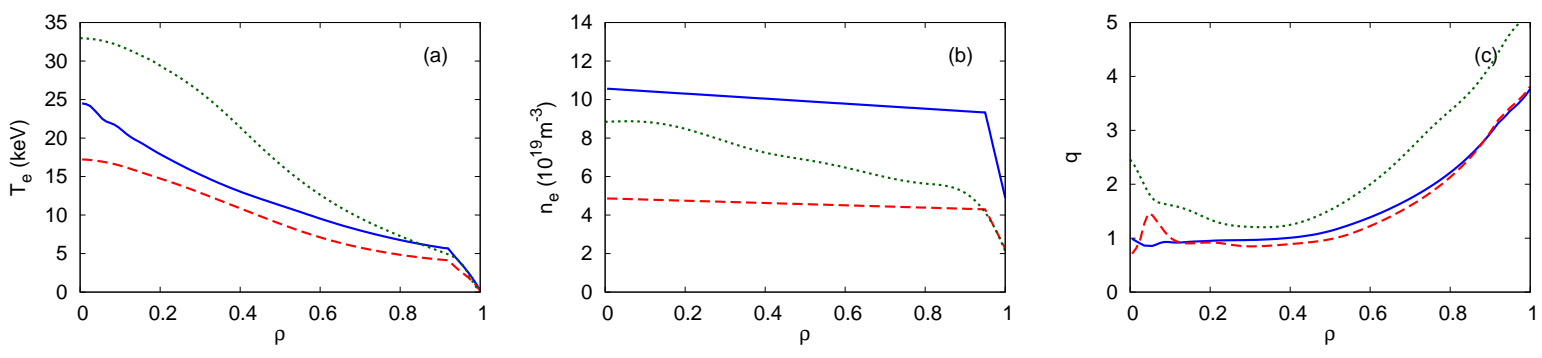

Figure 2. Radial profiles of electron temperature (a), density (b), and safety factor (c) at the end of the current flat-top of baseline (- - ), half-field (- - - ), and steady-state $(\ldots \ldots)$ scenarios.

Table 3. Plasma parameters on the $q=2$ surface, at the end of the current flat-top.

\begin{tabular}{lccc}
\hline & Case 1 & Case 6 & Case 8 \\
& $t=520 \mathrm{~s}$ & $t=820 \mathrm{~s}$ & $t=2450 \mathrm{~s}$ \\
\hline$\rho$ & 0.76 & 0.78 & 0.60 \\
$n_{e}\left(10^{19} / \mathrm{m}^{3}\right)$ & 9.58 & 4.40 & 6.47 \\
$T_{e}(\mathrm{keV})$ & 7.23 & 5.01 & 12.6 \\
$J_{\mathrm{bs}}\left(\mathrm{kA} / \mathrm{m}^{2}\right)$ & 74.4 & 33.0 & 215 \\
\hline
\end{tabular}

Table 4. Plasma parameters on the $q=3 / 2$ surface, at the end of the current flat-top.

\begin{tabular}{lccc}
\hline & Case 1 & Case 6 & Case 8 \\
& $t=520 \mathrm{~s}$ & $t=820 \mathrm{~s}$ & $t=2450 \mathrm{~s}$ \\
\hline$\rho$ & 0.64 & 0.68 & 0.49 \\
$n_{e}\left(10^{19} / \mathrm{m}^{3}\right)$ & 9.74 & 4.46 & 6.89 \\
$T_{e}(\mathrm{keV})$ & 8.95 & 6.06 & 16.9 \\
$J_{\mathrm{bs}}\left(\mathrm{kA} / \mathrm{m}^{2}\right)$ & 81.8 & 39.2 & 217 \\
\hline
\end{tabular}

respectively, to have a clear overview of the ECCD efficiency around the nominal toroidal injection angle $\beta=20^{\circ}$ and across a large fraction of the plasma cross section. The NTM stabilization efficiency has been evaluated at the $q=2$ and $q=3 / 2$ rational surfaces along the time evolution of the plasma discharges of cases 1, 2, 3 and 6 , for which NTM stabilization is a primary objective.

\subsection{ECCD efficiency at end of flat-top}

To evaluate ECCD efficiency at the end of the current flat-top, the injection angles have been varied in the range $25^{\circ} \leq \alpha \leq 65^{\circ}$, and $15^{\circ} \leq \beta \leq 25^{\circ}$, and the results for the LSM are shown in figure 3 and figure 4 , where the driven current $I_{\mathrm{cd}}$ and the peak current density $J_{\mathrm{p}}$ are represented as a function of the injection angles together with the driven current radial location $\langle\rho\rangle_{J}$. The current density $J_{\text {cd }}$ driven when aiming at the $q=2$ and $q=3 / 2$ rational surfaces is shown in figure 5 .

As expected, at a given radius $\rho$ the driven current $I_{\mathrm{cd}}$ increases with increasing 

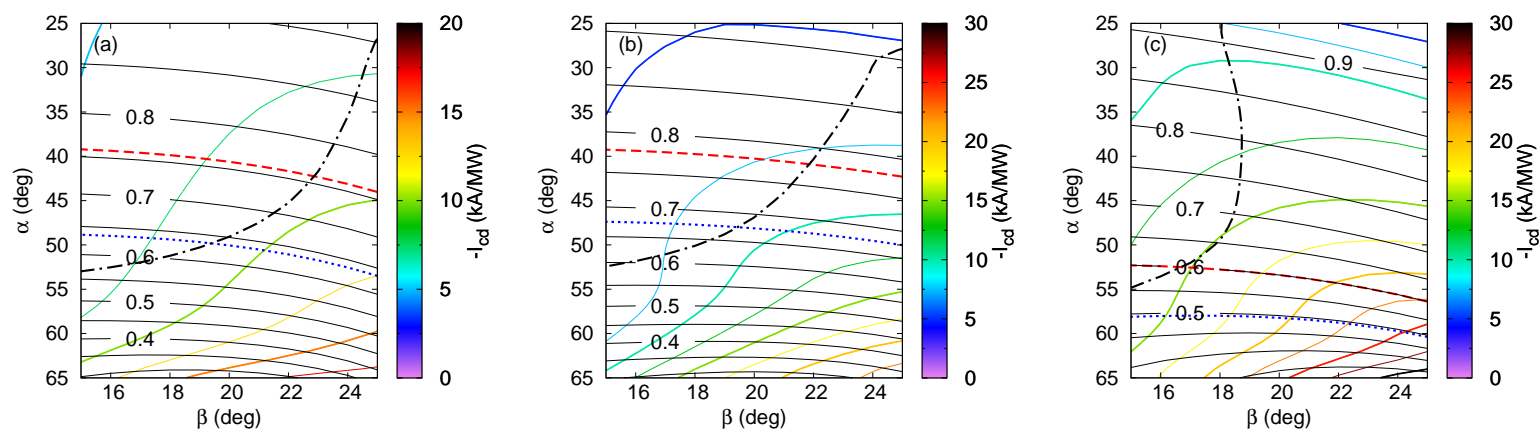

Figure 3. EC driven current per unit injected power (color contours) and radial location (labelled black contours) versus toroidal $\beta$ and poloidal $\alpha$ injection angles from LSM for baseline (a), half-field (b), and steady-state (c) scenarios at the end of the current flat-top. The injection angles required to drive current at the resonant surfaces $q=2(-\cdots$, red $)$ and $q=3 / 2(\ldots$, , blue) surfaces, and those giving a current profile width $w_{\mathrm{cd}}=4.2 \mathrm{~cm}(-\cdot-)$ are also shown.
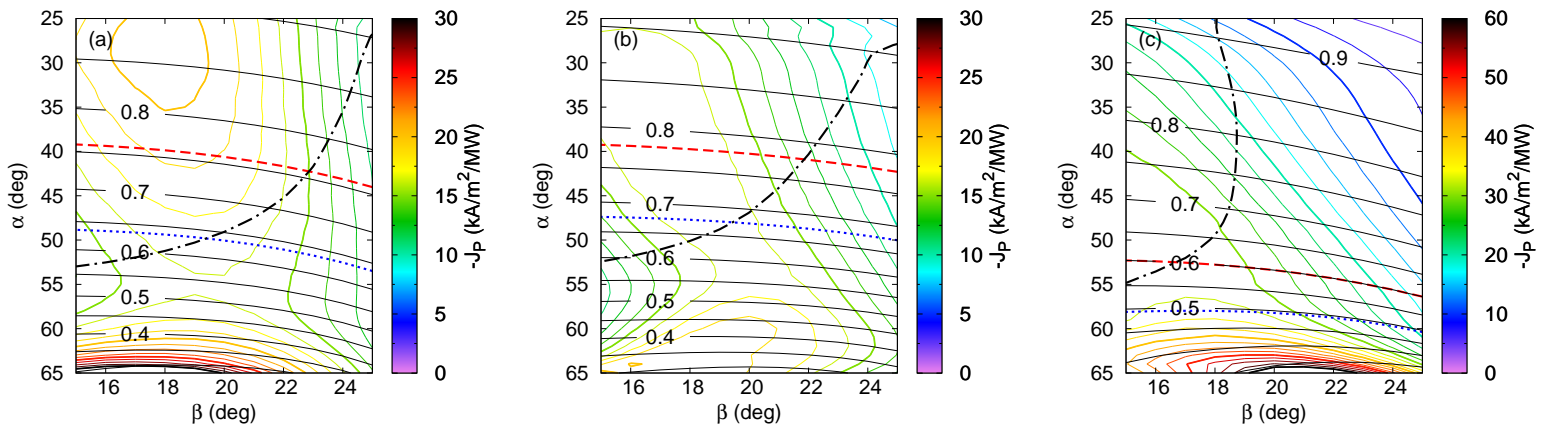

Figure 4. Same as figure 3 for the peak current density $J_{\mathrm{p}}$ per unit injected power.
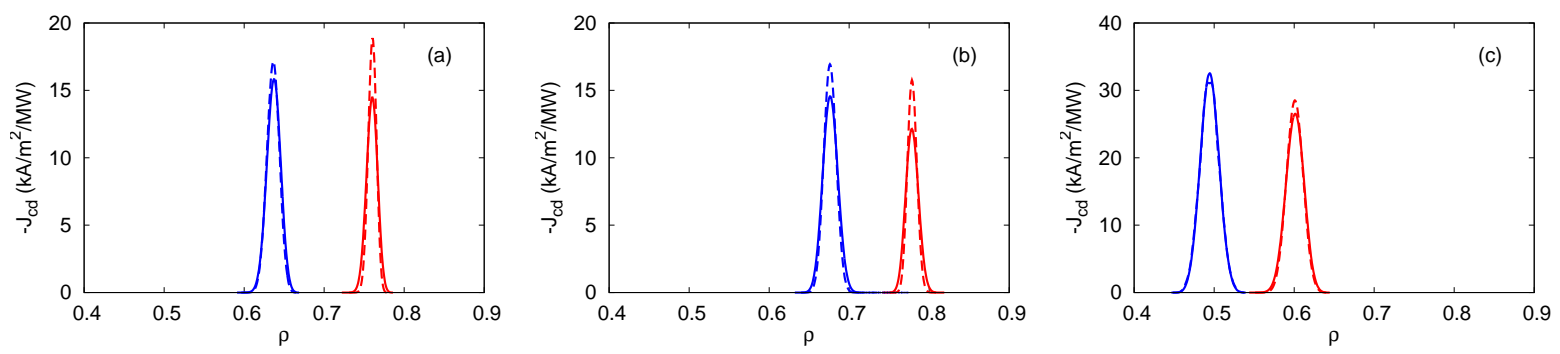

Figure 5. Driven current density profiles $J_{\text {cd }}$ when aiming at the $q=2$ (red) and $q=3 / 2$ (blue) surfaces (see tables 3 and 4 ) with $\beta=20^{\circ}$, from USM ( - ) or LSM (- - - ), at the end of the current flat-top of baseline (a), half-field (b), and steady-state (c) scenarios. 
toroidal injection angle, while the peak current density $J_{\mathrm{p}}$ has a weak dependence on $\beta$ with a broad maximum for both mirrors in the range $17^{\circ}<\beta<20^{\circ}$ in the radial region of interest for NTMs. Compared to the USM, for a given $\beta$ the LSM delivers approximately the same total current $I_{\text {cd }}$, but with larger peak current density $J_{\mathrm{p}}$ mainly due to the smaller size of the beam in the absorption region.

Cases 1 and 6 show a similar general behaviour, with rational surfaces approximately at the same radial location, maximum $J_{\mathrm{p}}$ at similar values of $\beta$, and the reference value of current profile width $w_{\mathrm{cd}}=4.2 \mathrm{~cm}$ is obtained at almost identical injection angles. CD efficiency is marginally higher at half-field than at full-field, due to the more advantageous $T_{\mathrm{e}} / n_{\mathrm{e}}$ ratio. The different shape of the $T_{\mathrm{e}}$ and $n_{\mathrm{e}}$ profiles for the Case 8 steady-state scenario, with even higher $T_{\mathrm{e}} / n_{\mathrm{e}}$ ratio in the outer plasma region, allows to obtain a driven current $I_{\mathrm{cd}}$ about 1.5 times higher than Cases 1 and 6 at given $\rho$. At the same time the $J_{\text {cd }}$ profiles are generally broader, so that a profile width $w_{\mathrm{cd}}=4.2 \mathrm{~cm}$ is achieved with smaller toroidal injection angles $\beta$, for which Doppler broadening is less effective, compared to the other two scenarios.

\subsection{Power requirements for NTM stabilization}

For the analysis performed here, the power required for NTM stabilization is estimated as the maximum between the values provided by the two criteria introduced in (2), and (3), $P_{\text {stab }}=\max \left(P_{\eta}, P_{\eta w}\right)$. The expressions used for the estimate of the NTM stabilization power involve the ratio between the peak value of the EC driven current density $J_{\text {cd }}$ and the bootstrap current density $J_{\mathrm{bs}}$ at the rational surface. For full consistency, the same definition has been used for $J_{\text {cd }}$ as that used for $J_{\text {bs }}$ in the different scenarios: $J_{\mathrm{cd}}=\left\langle\mathbf{J}_{\mathrm{cd}} \cdot \mathbf{B}\right\rangle / B_{\text {ref }}$, with $B_{\text {ref }}=\langle B\rangle$ for all the cases except Case 8 , in which $B_{\text {ref }}=5.3 \mathrm{~T}$.

Due to the dependence of $I_{\mathrm{cd}}$ and $J_{\mathrm{cd}}$ on the toroidal injection angle $\beta$ described in section 4.1, $P_{\eta w}$ decreases with increasing $\beta$, while $P_{\eta}$ increases. Thus, $P_{\text {stab }}=P_{\eta}$ for sufficiently large $\beta$, and $P_{\text {stab }}=P_{\eta w}$ at small toroidal injection angles, and as a consequence the power required at a given rational surface is minimized for a value of $\beta$ for which $w_{\text {cd }}=4.2 \mathrm{~cm}$. This dependence on the toroidal injection angle is shown in figure 6 for the full-field scenario. The minimum of $P_{\text {stab }}$ at the $q=2$ surface is obtained for an angle $\beta \simeq 21^{\circ}$, with $P_{\text {stab }}=6.5 \mathrm{MW}$, the optimal angle being slightly smaller at the $q=3 / 2$ surface $\left(\beta \simeq 19^{\circ}\right)$, with similar value of $P_{\text {stab }}$.

By comparing in figure 6 the results obtained for the same rational surface from the USM or from the LSM, it can be noted that the power requirement is essentially the same for the two mirrors at small $\beta$ values: as long as the driven current profile width $w_{\text {cd }}$ remains below the threshold $w_{\mathrm{cd}}=4.2 \mathrm{~cm}$ for both mirrors the criterion $P_{\text {stab }}=P_{\eta w}$ holds, so that the required power is determined by the total driven current $I_{\text {cd }}$ which is very little influenced by the slightly different launching position and beam shape of the two mirrors. On the contrary at larger $\beta$ values, when $w_{\mathrm{cd}}>4.2 \mathrm{~cm}, P_{\mathrm{stab}}=P_{\eta}$ so that the peak driven current density value, which depends on $w_{\mathrm{cd}}$ for a given $I_{\mathrm{cd}}$ value, 

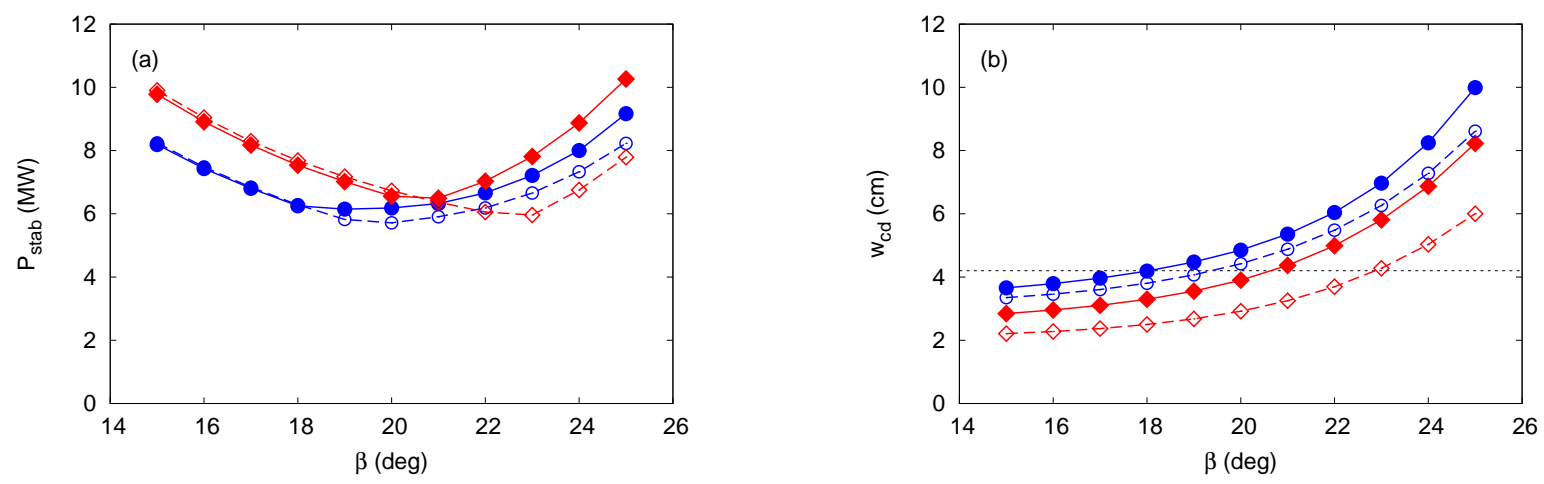

Figure 6. Power $P_{\text {stab }}$ required for NTM stabilization (a) and driven current profile width $w_{\mathrm{cd}}$ (b) for USM ( $\longrightarrow$ ) and LSM ( - - -) aimed at $q=2(\diamond$, red) and $q=3 / 2$ $(\mathrm{O}$, blue) surfaces, as a function of the toroidal launching angle $\beta$. Case $1, t=520 \mathrm{~s}$.

becomes important to determine the power requirement.

Care must then be used when interpreting these results: the value $\beta_{\min }$ for which the minimum power $P_{\mathrm{s}, \min }$ is achieved depends on the choice made in the design of the launching system. The difference between USM and LSM here is a direct consequence of their different beam widths at the resonance: for any given scenario, a narrowing of the beam allows a reduction of $P_{\mathrm{s}, \min }$, but the lower $P_{\mathrm{s}, \min }$ value is achieved at a larger $\beta_{\min }$, which is anyway limited by engineering constraints. At the same time, any additional effect which may broaden the deposition profile [17] would go in the opposite direction, moving the minimum at lower $\beta_{\min }$ values and increasing the minimum required power.

A similar trend is found for the other cases, as shown in tables 5 and 6 , which summarize the values obtained in the three scenarios for both the steering mirrors. The half-field scenario requires lower power levels, about 3.2 MW at the $q=2$ surface for the USM, due mainly to lower bootstrap current, and also to larger $T_{\mathrm{e}} / n_{\mathrm{e}}$ ratio. The high power required in the steady-state scenario despite the high CD efficiency is easily explained with the large bootstrap current present in this scenario. It can also be noted that the minimum $P_{\mathrm{s}, \mathrm{min}}$ is reached at a smaller angle $\beta$ than the other scenarios due to the larger profile width $w_{\mathrm{cd}}$ obtained at a given $\beta$. The optimal toroidal injection angle minimizing $P_{\text {stab }}$ is in the range $16^{\circ} \leq \beta_{\text {min }} \leq 23^{\circ}$, depending on scenario, steering mirror and rational surface considered. So the optimal value $\beta_{\min }$ changes by as much as $7^{\circ}$ depending on the conditions, but $P_{\text {stab }}$ always shows quite a broad minimum at $\beta_{\min }$, and the requirements at the nominal value $\beta=20^{\circ}$ are only marginally larger.

In addition $P_{\text {stab }}$ has been evaluated along the whole time history of the different scenarios. At each time step the poloidal injection angle necessary to aim at the timeevolving position of the rational surfaces has been evaluated. The power $P_{\text {stab }}$ has then been computed using the $J_{\mathrm{cd}}$ and $w_{\mathrm{cd}}$ values provided by the GRAY code and the time-dependent $J_{\mathrm{bs}}$ value. The results are shown in figure 7 : the stabilization power is almost constant during the current flat-top at levels lower than the power available from a single set of steering mirrors $P_{\mathrm{EC}}=13.3 \mathrm{MW}$, and it drops at even lower levels 
Table 5. Power $P_{\mathrm{s}, \min }$ required at the end of flat-top for NTM stabilization, for USM and LSM at the optimal angle $\beta_{\min }$.

\begin{tabular}{lllll}
\hline & \multicolumn{2}{c}{$q=2$} & \multicolumn{2}{c}{$q=3 / 2$} \\
& $\begin{array}{l}P_{\mathrm{s}, \min } \\
(\mathrm{MW})\end{array}$ & $\begin{array}{l}\beta_{\min } \\
\left.{ }^{\circ}\right)\end{array}$ & $\begin{array}{l}P_{\mathrm{s}, \min } \\
(\mathrm{MW})\end{array}$ & $\begin{array}{l}\beta_{\min } \\
\left.{ }^{\circ}\right)\end{array}$ \\
\hline Case 1, USM & 6.5 & 21 & 6.1 & 19 \\
Case 1, LSM & 6.0 & 23 & 5.7 & 20 \\
Case 6, USM & 3.2 & 19 & 3.1 & 18 \\
Case 6, LSM & 3.0 & 22 & 2.8 & 20 \\
Case 8, USM & 8.3 & 16 & 7.2 & 16 \\
Case 8, LSM & 7.8 & 17 & 7.6 & 17 \\
\hline
\end{tabular}

Table 6. Power $P_{\mathrm{s}, 20}$ required at the end of current flat-top for NTM stabilization and current profile width $w_{\mathrm{cd}, 20}$, for USM and LSM at $\beta=20^{\circ}$.

\begin{tabular}{lllll}
\hline & \multicolumn{2}{c}{$q=2$} & \multicolumn{2}{c}{$q=3 / 2$} \\
& $\begin{array}{l}P_{\mathrm{s}, 20} \\
(\mathrm{MW})\end{array}$ & $\begin{array}{l}w_{\mathrm{cd}, 20} \\
(\mathrm{~cm})\end{array}$ & $\begin{array}{l}P_{\mathrm{s}, 20} \\
(\mathrm{MW})\end{array}$ & $\begin{array}{l}w_{\mathrm{cd}, 20} \\
(\mathrm{~cm})\end{array}$ \\
\hline Case 1, USM & 6.6 & 3.9 & 6.2 & 4.9 \\
Case 1, LSM & 6.7 & 2.9 & 5.7 & 4.4 \\
Case 6, USM & 3.2 & 4.5 & 3.2 & 5.3 \\
Case 6, LSM & 3.2 & 3.3 & 2.8 & 4.4 \\
Case 8, USM & 9.4 & 6.3 & 8.0 & 6.6 \\
Case 8, LSM & 8.8 & 5.8 & 8.1 & 6.7 \\
\hline
\end{tabular}

during the current ramp phases. However, large peaks with $P>P_{\mathrm{EC}}$ show up at the $\mathrm{L}-\mathrm{H}$ and $\mathrm{H}-\mathrm{L}$ transitions. The same occurs in the half-field scenario, while the late $\mathrm{H}-\mathrm{L}$ transition of Case 2, at $t=743 \mathrm{~s}$ when the plasma parameters are all reduced, allows to remain within the limits of available power. It should be noted here that the appearance of these spikes are a direct consequence of the sharp variation observed in the plasma temperature across the L-H and $\mathrm{H}-\mathrm{L}$ transitions, while a slow time evolution is imposed to the density. For this reason a more detailed, possibly fully-predictive, simulation of the transitions would be desirable to support more conclusive comments on these phases.

About the the criteria introduced in (4) and (5) and used throughout this analysis, one should note that these criteria provide the power value sufficient to stabilize an island at any stage of its growth towards saturation, assuming that the plasma parameters do not change significantly during the time required for the stabilization. As a consequence, with a pre-emptive method, in which MHD activity is suppressed before the island can develop, lower power values will be required. Moreover, even with a non-preemptive method, a power lower than $P_{\text {stab }}$ may be sufficient during the current ramp-down because the plasma parameters decay during the island's growth time, possibly reducing 


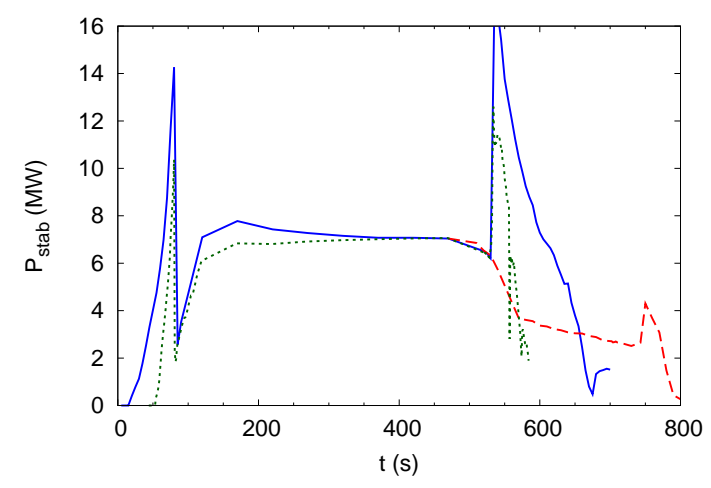

Figure 7. Power $P_{\text {stab }}$ required for NTM stabilization at the $q=2$ surfaces during the plasma discharge of Cases $1(-), 2(---)$, and $3(\cdots \cdots)$. Injection from the USM with $\beta=20^{\circ}$.
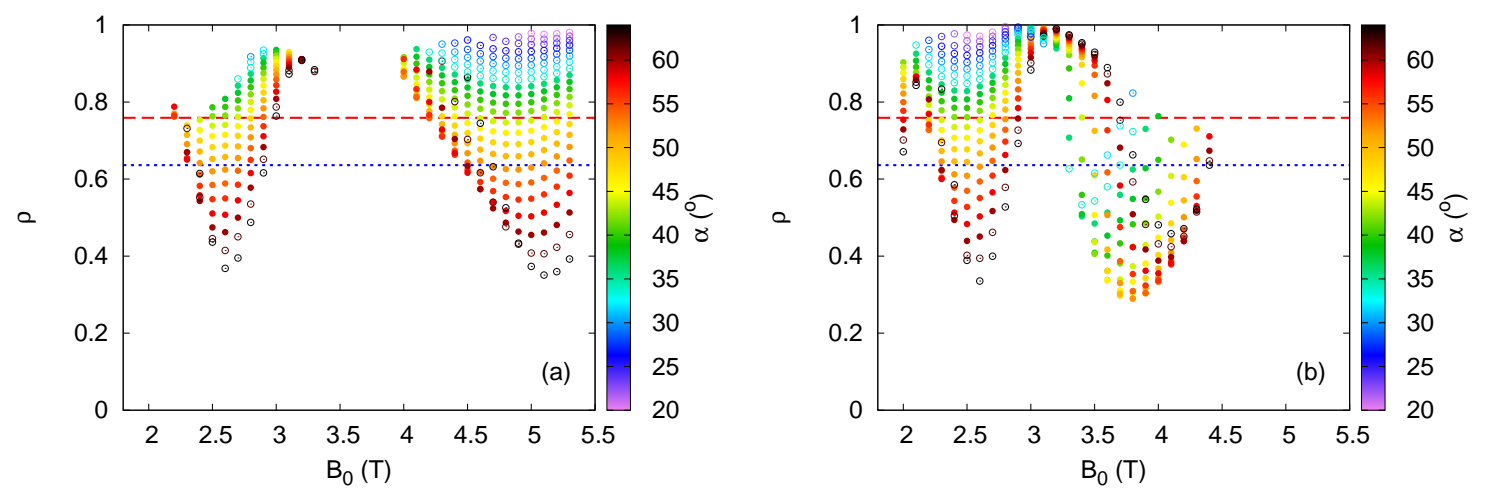

Figure 8. Average power deposition radius $\rho$ for O-mode (a) and X-mode (b) polarized wave injection from USM as a function of the central vacuum magnetic field strength $B_{0}$. Full symbols for $36^{\circ} \leq \alpha \leq 60^{\circ}$. Color coding indicates the angle $\alpha$ used to reach a given radius $\rho$. Radial location of $q=2(-\ldots$, red) and $q=3 / 2(\ldots$, , blue) are shown as reference. Case 1 scenario at $t=520 \mathrm{~s}$, scaled according to (8). Only cases with more than $95 \%$ absorbed power are represented.

its growth rate.

\subsection{Intermediate magnetic field values}

An interesting issue is to evaluate the H\&CD efficiency from the UL when ITER will be operated at gradually increasing magnetic field values, from the first operations at halffield up to the full nominal field of $5.3 \mathrm{~T}$. The intermediate field cases have been studied by rescaling the full-field case 1 with unchanged safety factor $q$, normalized pressure $\beta_{p}$, and Greenwald fraction $n / n_{\mathrm{G}}$. If the magnetic field is scaled by a factor $\gamma$, plasma current $I_{\mathrm{p}}$, pressure $p$, electron density $n_{\mathrm{e}}$ and temperature $T_{\mathrm{e}}$ are scaled according to $[4,5]$ :

$$
\frac{I_{\mathrm{p}}^{\prime}}{I_{\mathrm{p}}}=\frac{n_{\mathrm{e}}^{\prime}}{n_{\mathrm{e}}}=\frac{T_{\mathrm{e}}^{\prime}}{T_{\mathrm{e}}}=\left(\frac{p^{\prime}}{p}\right)^{1 / 2}=\frac{B^{\prime}}{B}=\gamma
$$


We note here that ECCD efficiency is roughly proportional to the ratio $T_{\mathrm{e}} / n_{\mathrm{e}}$, and so at first approximation it is expected to remain constant when $B$ is scaled.

The magnetic field $B_{0}$ at the vessel centre has been varied in the range $2 \mathrm{~T} \leq B_{0} \leq$ $5.3 \mathrm{~T}$ with steps of size $\delta B=0.1 \mathrm{~T}$. For every scaled plasma, both O-mode and $\mathrm{X}$ mode injection has been considered from the two steering mirrors, with a fixed toroidal launching angle $\beta=20^{\circ}$ and a poloidal angle $\alpha$ varying in the range $20^{\circ} \leq \alpha \leq 65^{\circ}$, which is wider than the actual steering capability $\Delta \alpha_{\text {steer }} \simeq 24^{\circ}$ permitted by the engineering constraints. The exact limits of the steering can be adjusted by a few degrees in the finalization of the design, by a rigid rotation of the whole steering mirror assembly. Figure 8 shows the average deposition radii reachable with the poloidal steering capabilities of the system, with at least $95 \%$ absorption of the injected power. The two plots allow comparing how this radial range evolves for O-mode and X-mode injection from the USM when the magnetic field strength $B_{0}$ is varied.

The range of magnetic field values for which absorption can take place at the $q=3 / 2$ and $q=2$ surfaces is quite extended, being $2.3 \mathrm{~T} \leq B_{0} \leq 3 \mathrm{~T}$ for the second harmonic, and $B_{0} \geq 4.2 \mathrm{~T}$ for the first harmonic. As expected, the widest radial range coverage, $0.45 \leq \rho \leq 0.85$, is achieved for a magnetic field close to the nominal value $5.3 \mathrm{~T}$, where the system has been optimized, with O-mode absorption at the first harmonic resonance, while the cut-off layer inhibits direct X-mode absorption at the first harmonic resonance for magnetic fields $B_{0} \geq 4.5 \mathrm{~T}$. A wide radial range can be covered also around the half-field value $B_{0} \simeq 2.65 \mathrm{~T}$ both with $\mathrm{O}$-mode and $\mathrm{X}$-mode absorption at the second harmonic. However, O-mode absorption at the second harmonic is weaker than X-mode, so that $\mathrm{O}$-mode absorption profiles are broader and the outer radii $\rho \geq 0.8$ can be reached retaining full absorption only with $\mathrm{X}$-mode. Another feature of $\mathrm{X}$-mode injection is that it allows to reach the inner radii $0.3 \lesssim \rho \lesssim 0.7$ also for $3.2 \mathrm{~T} \leq B_{0} \leq 4.5 \mathrm{~T}$ when both the fundamental and the second harmonic resonance are outside the plasma volume, exploiting the down-shifted first harmonic resonance of hot electrons on the high field side of the cold resonance.

Current drive efficiency has been evaluated for every scaling factor and for every injection angle $\alpha$. The results are shown in figure 9 for a beam aimed at the $q=3 / 2$ or $q=2$ surface. Within each of the two $B_{0}$ ranges corresponding to first and second harmonic interaction, the driven current $I_{\mathrm{cd}}$ increases with decreasing $B$ so that the highest efficiency is achieved at the lower end of the two $B_{0}$ intervals. Considering for example O-mode at the first harmonic, the current driven for $B_{0}=4.5 \mathrm{~T}$ is about $25 \%$ higher than the nominal case $B_{0}=5.3 \mathrm{~T}$. Such a trend can be explained by the fact that when $B_{0}$ is reduced the resonance shifts toward the high field side of the plasma, lowering the fraction of trapped electrons at the absorption region. On top of this the bootstrap current $J_{b s}$ is expected to scale as $B_{0}$, reducing the power requirements for NTM control at $B_{0}<5.3 \mathrm{~T}$ even further.

At $B_{0} \lesssim 3 \mathrm{~T}$, second harmonic X-mode shows a CD efficiency similar to O-mode at the first harmonic, of the order of $10 \mathrm{kA} / \mathrm{MW}$, while the efficiency of O-mode at second harmonic is drastically lower. Some current can be driven at the $q=3 / 2$ surface with 


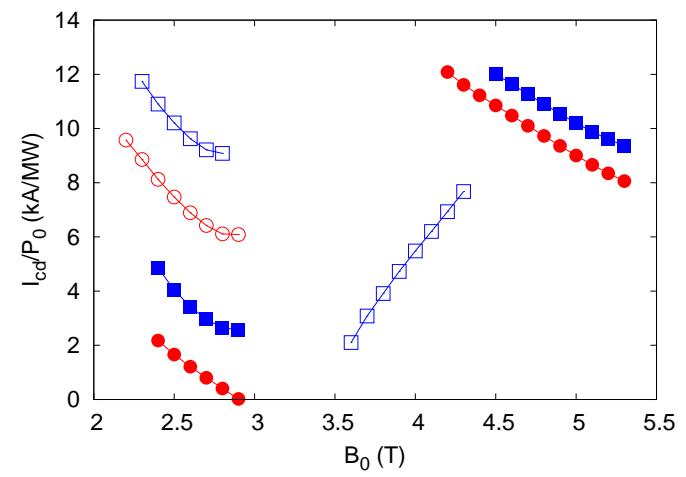

Figure 9. Current drive efficiency at intermediate values of the magnetic field $B_{0}$ for OM (full symbols) and XM (open symbols) injected from the USM aimed at the $q=2$ $(\mathrm{O}$, red) and $q=3 / 2(\square$, blue) surfaces. Same case and scaling of figure 8 .

X-mode injection even for $3.5 \mathrm{~T} \leq B_{0} \leq 4.5 \mathrm{~T}$, with higher $I_{c d}$ for increasing $B_{0}$, but this configuration might be difficult to control. In fact, for these values of $B_{0}$ the cold second harmonic resonance is too far on the low field side to be crossed directly, and a cut-off layer is still present on the high field side: the beam is strongly deflected by the cut-off and then absorbed at down-shifted second harmonic resonance.

\subsection{Steering requirements}

The design of the ITER EC upper launcher is particularly challenging, because to fulfil all its functions it must cover a wide radial range, and to focus its power on a target a few centimetres wide from a distance of the order of two meters. For this reason, a brief analysis has been performed to assess the requirements on the steering range, on the aiming accuracy, and on the handling of multiple beams.

In figure 10, the poloidal angle $\alpha$ necessary to aim the USM at the two rational surfaces is plotted versus the time history of the plasma discharge, for the reference toroidal angle value $\beta=20^{\circ}$. The range $\Delta \alpha=24^{\circ}$ allowed by the steering mechanism is sufficient to aim at both $q=2$ and $q=3 / 2$ surfaces in all the relevant phases of all the scenarios, however there is small margin: the range must be centered accurately to allow use of the UL also during transient events and for the other desirable tasks, such as current profile tailoring or sawtooth control.

The steering sensitivity has been assessed by computing the derivative of the driven current radial location $\rho$ with respect to the injection angle $\alpha$. The results are very similar for the two steering mirror, however the slightly different injection geometry makes the USM more sensitive to changes of the poloidal injection angle, so that a tighter tolerance on $\alpha$ is necessary but a smaller steering range is sufficient to cover the same radial extent.The values obtained at the end of the current flat-top are shown in figure 11, and are in the range $0.01 \mathrm{deg}^{-1} \lesssim|\mathrm{d} \rho / \mathrm{d} \alpha| \lesssim 0.03 \mathrm{deg}^{-1}$.

From the steering sensitivity we can derive the steering accuracy required to properly aim a beam at a rational surface. Let's express the radial shift experienced 


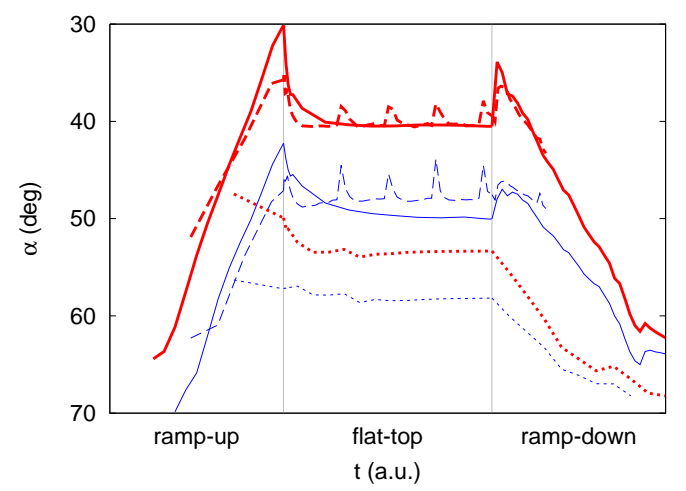

Figure 10. Poloidal injection angle $\alpha$ necessary to aim at the $q=2$ (thick, red) and $\mathrm{q}=3 / 2$ (thin, blue) surfaces, along the time history of the baseline $(-)$, half-field $(---)$, and steady-state $(\cdots \cdots)$ scenarios. Injection from the LSM with $\beta=20^{\circ}$. Time axis is not in scale.

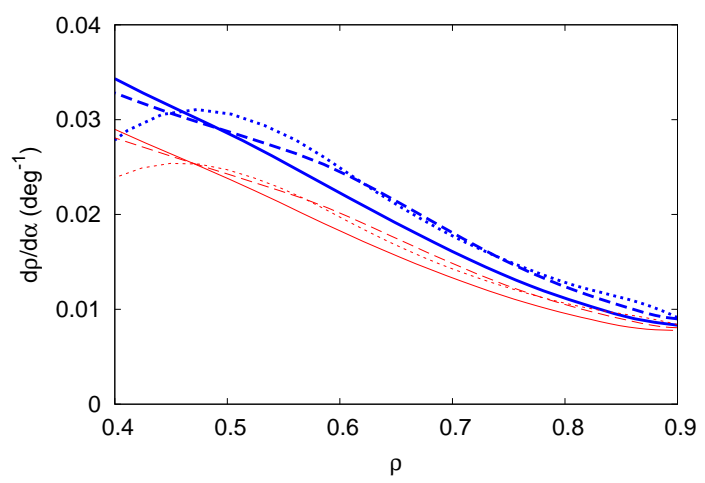

Figure 11. Steering sensitivity $|\mathrm{d} \rho / \mathrm{d} \alpha|$ of USM (thick, blue) and LSM (thin, red) at the end of the current flat top of the baseline (_- ), half-field (- - - ), and steady-state $(\cdots \cdots)$ scenarios for $\beta=20^{\circ}$.

by the driven current profile under a change of the injection angle $\alpha$ by $1^{\circ}$ as $\delta_{1}=$ $a \cdot 1^{\circ}|\mathrm{d} \rho / \mathrm{d} \alpha|$, where $a=2 \mathrm{~m}$ is the plasma minor radius. Its value varies in the range $0.02 \mathrm{~m} \lesssim \delta_{1} \lesssim 0.06 \mathrm{~m}$ depending on the radial position, and it increases with decreasing $\rho$, so that it is larger on the $q=3 / 2$ surface than on the $q=2$ surface. Considering that both the optimal driven current profile width and the marginal island width are of the order of $w_{\mathrm{cd}} \simeq 0.04 \mathrm{~m}$, an accuracy of $\Delta \alpha_{\text {acc }} \simeq 0.7^{\circ}$ is necessary at the innermost radii $\rho<0.5$ and less stringent requirements moving outwards, which is compatible with the capabilities expected for the steering mechanism.

The steering sensitivity can also be useful as a guideline in the design of the launcher optics, when the effect of having multiple beams launched by a single mirror is taken into account. Let's consider two Gaussian profiles of width $w_{\mathrm{cd}}$ : their sum starts to show two distinct maxima when they are separated by an amount $\delta>\delta_{\mathrm{m}}=w_{\mathrm{cd}} / \sqrt{2}$. This means that two beams designed to deliver perfectly overlapped current profiles under ideal conditions, become misaligned when their injection angle deviates from the intended value by more than $\Delta \alpha_{\mathrm{m}}=w_{\mathrm{cd}} /(\sqrt{2} a|\mathrm{~d} \rho / \mathrm{d} \alpha|)$. This figure can be thought 
as the maximum deviation allowed for the actual design from an ideal setup, able to keep all the beams overlapped along the steering range, before the performance starts to degrade, and it has a typical value of $\Delta \alpha_{\mathrm{m}} \sim 1^{\circ}$ for both USM and LSM in the baseline scenario at the end of the current flat-top.

\section{Conclusions}

A variety of ITER scenarios, covering the different phases of ITER operation, have been analyzed along all their time history to obtain an overview of the UL performance that can be expected with injection geometries consistent with the present design of the launcher, and considering a reasonable range of injection angle around it to provide a basis for future design optimization.

The power required for NTM stabilization has been evaluated through simple criteria over a range of toroidal injection angles at selected time slices, and for selected angles over the full time history of the plasma discharges, in order to identify the optimal angles and the critical phases in the discharge respectively. The analysis confirms the nominal toroidal injection angle $\beta=20^{\circ}$ to be very close to the optimal value. According to the criteria used, the power required for NTM stabilization, $P_{\text {stab }}<7 \mathrm{MW}$ for the baseline scenario at the current flat-top, is lower than the $20 \mathrm{MW}$ available with a safe margin. Still, a verification with a more detailed modelling of the transient phases of the discharges and of the NTM time evolution would be desirable.

The achievable steering range and accuracy appears adequate to fulfil the foreseen tasks, and the steering sensitivity assessment can be used as a basis to optimize the strategies for NTM tracking and control and to drive the finalization of the UL design. The impact of physical processes leading to possible modifications of the ECCD profiles with respect to standard beam-tracing predictions has been investigated as a complement of the present analysis [17]. EC heating and current drive from the UL has also demonstrated to be efficient for a relatively extended range of magnetic field values, giving confidence about the possibility to keep a good performance also in scenarios with magnetic fields different from the nominal ones.

The analysis presented here represents a basis for future more realistic and up to date scenarios modelling, which will take into account the EC heating and CD sources and their use for multiple tasks in a consistent way.

\section{Acknowledgments}

We wish to thank O. Sauter, T. Goodman, and the CRPP team for the fruitful discussions and the support provided in the preparation of the equilibrium data used for this analysis. This work was supported by Fusion for Energy under the grant contract F4E-2010-GRT-161. The views and opinions expressed herein reflect only the author's views. Fusion for Energy is not liable for any use that may be made of the information contained therein. 


\section{References}

[1] Henderson M A et al 2008 Nucl. Fusion 48054013

[2] Henderson M et al 2014 ITER EC H\&CD Targeted Functional Capabilities Phys. Plasmas (submitted)

[3] Ramponi G, Farina D, Henderson M A, Poli E, Saibene G and Zohm H 2007 Fusion Sci. Technol. 52 193-201

[4] Ramponi G, Farina D, Henderson M A, Poli E, Sauter O, Saibene G, Zohm H and Zucca C 2008 Nucl. Fusion 48054012

[5] Farina D, Henderson M, Figini L, Ramponi G and Saibene G, 2012 Nucl. Fusion 52033005

[6] Farina D, Henderson M, Figini L and Saibene G 2014 Phys. Plasmas 21061504

[7] Pereverzev G V 1998 Phys. Plasmas 53529

[8] Maj O, Mariani A, Poli E, Farina D 2013 Phys. Plasmas 20042122

[9] Harvey R W, McCoy M G and Kerbel G D 1989 Phys. Rev. Lett. 62426

[10] Farina D 2007 Fusion Sci. Technol. 52 154-160

[11] Marushchenko N B, Erckmann V, Hartfuss H J, Hirsch M, Laqua H P, Maassberg H and Turkin Y 2007 Plasma Fusion Res. 2 S1129

[12] Prater R et al 2008 Nucl. Fusion 48035006

[13] Figini L, Decker J, Farina D, Marushchenko N B, Peysson Y, Poli E, Westerhof E, and ITM-TF contributors 2012 EPJ Web of Conferences 3201011

[14] Moro A et al 2014 AIP Conference Proceedings 1580 550-553

[15] Rutherford P H 1973 Phys. Fluids 161903

[16] Sauter O et al 1997 Phys. Plasmas 41654

[17] Poli E et al 2015 Nucl. Fusion 55013023

[18] Zohm H, Gantenbein G, Leuterer F, Maraschek M, Poli E, Urso L and the ASDEX Upgrade Team 2007 Plasma Phys. Control. Fusion 49 B341

[19] Sauter O, Henderson M A, Ramponi G, Zohm H and Zucca C 2010 Plasma Phys. Control. Fusion 52025002

[20] Bertelli N, De Lazzari D and Westerhof E 2011 Nucl. Fusion 51103007

[21] Parail V et al 2013 Nucl. Fusion 53113002 